\title{
Assessing the Internal Structure of Hollow Trees Using GPR and Microwave Tomography
}

\author{
Fabio Tosti", Gianluca Gennarelli†, Livia Lantini ${ }^{*}$, Ilaria Catapano ${ }^{\dagger}$, Lilong Zou*, Francesco Soldovieri†, Amir M. \\ Alani* $^{*}$ \\ *School of Computing and Engineering, University of West London (UWL), St Mary's Road, Ealing, London W5 5RF, UK \\ 'Institute for Electromagnetic Sensing of the Environment (IREA-CNR), National Research Council, Via Diocleziano 328, 80124, \\ Naples, Italy \\ Fabio.Tosti@uwl.ac.uk; gennarelli.g@irea.cnr.it; Livia.Lantini@uwl.ac.uk; catapano.i@irea.cnr.it; Lilong.Zou@uwl.ac.uk; \\ soldovieri.f@irea.cnr.it; Amir.Alani@uwl.ac.uk
}

\begin{abstract}
Trees and woodlands are nowadays threatened by variety of aggressive diseases and fungal infections. As a result, internal decays in trees, can lead to the creation of cavities and large holes inside the trunks, which in turn can seriously undermine the stability and the integrity of the tree. In this regard, ground-penetrating radar (GPR) has recently proven to be an effective non-destructive testing (NDT) method, with the potential of providing information about the internal structure of trees. However, the particular shape of tree trunks prevents the use of traditional data processing techniques, and only limited information can be collected for tree health assessment purposes. This study shows the potential of GPR enhanced by a microwave tomography inversion approach in detecting tree cavities and hollows. A hollow tree was investigated by performing a set of circular GPR scans, and the internal structure of the trunk was reconstructed via tomographic imaging. The achieved results were validated by way of comparison with real sections cut from the tree and prove the validity of the proposed methodology in identifying the dimension and shape of cavities and hollows in tree trunks.
\end{abstract}

Keywords-ground-penetrating radar (GPR); hollow trees; microwave tomography; NDT; tree health monitoring

\section{INTRODUCTION}

Health monitoring and conservation of woodlands and forests are vital to ensure the survival of ecosystems and the maintenance of climate conditions. At present, the protection of natural heritage is of crucial importance since the incidence of emerging infectious diseases (EIDs) is continuously increasing [1], often leading to the death of entire forests over a short period of time. Specifically, some particular bacteria and fungi attack the trees by entering wounds or damage created by the action of external agents, such as excavation procured by birds, insects, or fire [2]. These infections, such as the heart rot disease [3], can escalate into the decomposition of heartwood, and subsequently

Acknowledgements: The authors would like to express their sincere thanks and gratitude to the following trusts, charities, organisations and individuals for their generosity in supporting this project: Lord Faringdon Charitable Trust, The Schroder Foundation, Cazenove Charitable Trust, Ernest Cook Trust, Sir Henry Keswick, Ian Bond, P. F. Charitable Trust, Prospect Investment Management Limited, The Adrian Swire Charitable Trust, The John Swire 1989 Charitable Trust, The Sackler Trust, The Tanlaw Foundation, and The Wyfold Charitable Trust. This paper is dedicated to the memory of Jonathan West, a friend, a colleague, a forester, a conservationist and an environmentalist who died following an accident in the woodland that he loved. forming cavities and hollows into the trunks $[4,5]$. To this effect, it is important to note that a tree hollow does not necessarily compromise the survival of the plant [2], as heartwood is composed of dead tissues, and consequently it does not contribute to the bio-physiological processes of the tree [6]. In fact, the tree can continue to increase in size [7], as the distribution and storage of nutrients takes place in the sapwood [6]. However, the decomposition of the heartwood, whose primary function is to provide structural support [6], and potential formation of hollows can affect heavily the stability of the tree. Strength of the stem is reduced by the presence of cavities and hollows, resulting in trees being structurally weaker and more likely to break under the action of external forces, such as wind load [8]. This can create dangerous scenarios, especially in case of hollow trees located in public parks or alongside roads, where breakage can be harmful to people and properties $[8,9]$.

Within this framework, the identification of the early stage symptoms of the above-mentioned infections is essential to identify affected trees and implement control measures. This is however a challenging task, as first symptoms of fungal infections are mostly located in the innermost core of the trunk, and cannot be recognised by visual inspection [10]. In addition to this, it is important to monitor key features in hollow trees, such as the thickness of the bark, to ensure that critical conditions for the structural stability of trees have not been reached.

Core drilling is still one of the most used methods for decay detection, despite of its major intrusiveness. Drilling damages the tree trunk, and leads potentially to new infections. To this extent, the use of non-destructive testing (NDT) methods, such as ultrasound tomography [11], electrical resistivity tomography (ERT) [12], X-ray tomography [13] and infrared thermography [14] has been investigated in this area of endeavour. Microwave tomography is classified as an electromagnetic (EM) method and has provided good results in tree assessment applications [15-17]. Furthermore, ground-penetrating radar (GPR) has proven to be a suitable and powerful method in forestry engineering applications [18]. Recent research has shown GPR potential in tree trunks monitoring $[18,19]$ and tree root system investigations [20-23].

It is important to consider that, given the cylindrical-like shape of tree trunks and the random location and size of internal 
anomalies, traditional GPR signal processing techniques do not represent a viable solution to the problem of decay detection. On the other hand, tomographic approaches have proven to be effective for investigation of trees [15-17] and other cylindricalshaped objects $[24,25]$. In addition to this, numerical simulation of GPR signal has been used for interpreting the shape and position of tree trunks' internal decays $[26,27]$.

\section{AIM AND OBJECTIVE}

The aim of this study is to implement a viable and robust GPR data processing methodology for the assessment of the main structural features of hollow trees (i.e., thickness of the structural solid layer and potential cracks).

To achieve this aim, the feasibility of a microwave tomography inversion approach is analysed in the present research.

Specifically, GPR data from a hollow tree were collected, and pre-processing was applied to create the input dataset for application of the microwave tomographic approach. The tree was eventually felled and a few sections were cut for validation purposes.

\section{METHODOLOGY}

\section{A. The Survey Technique}

The survey was carried out in Gunnersbury Park, Ealing, London (United Kingdom). A matured tree containing a cavity was examined. The presence of a tree hollow was known based on previous investigations.

Circular measurements were collected parallel to the ground, spaced $0.1 \mathrm{~m}$ from one another. The investigated area was $1.5 \mathrm{~m}$ height, and it was covered by 15 scans (Fig. 1). The first scan was taken at the bottom of the tree $(h=0 \mathrm{~m})$, whereas the last one was collected at the top section $(h=1.5 \mathrm{~m})$.

The radius of the circle approximating the contour of the tree sections was derived indirectly from the measured circumference and the average radius of the trunk was equal to $0.63 \mathrm{~m}$.

A $2 \mathrm{GHz}$ Aladdin hand-held antenna system, manufactured by IDS GeoRadar (Part of Hexagon), was used for testing purposes. The time-step was equal to $\Delta t=6.25 \cdot 10^{-11} \mathrm{~s}$ and the spatial step of the measuring wheel was $\Delta s=1 \mathrm{~cm}$.

Subsequently, the tree was felled and the investigated part of the trunk was cut into $0.2 \mathrm{~m}$ thick slices, which were used for validation purposes.

\section{B. Data Processing Framework: the Microwave Tomography Inversion Approach}

The microwave tomography inversion approach adopted in this study exploits a linearised model of the EM scattering based on the Born Approximation (BA) [28, 29]. In this regard, it is well-known that BA allows for a quantitative reconstruction only if the targets in the scenario are weak scatterers.

However, if the weak scattering assumption is no longer valid, it is still possible to obtain reliable information regarding the presence, position and approximate shape of targets [30].

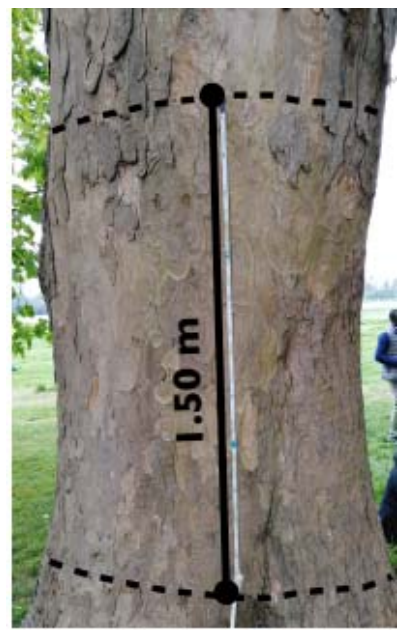

Fig. 1. The investigated tree.

In this study, a linear inverse scattering approach based on the BA is adopted under a multi-monostatic/multi-frequency configuration. A 2D scenario is considered, consisting of the cross-section of a tree trunk where the measurement points $\boldsymbol{r}_{m}$ are located at a fixed height. The investigated region $D$, which corresponds to the trunk section, is assumed homogeneous with a constant relative dielectric permittivity $\varepsilon_{b}$. The presence of targets in $D$ is described by the contrast function $\chi$, which accounts for the difference between the targets' permittivity and the permittivity of the background medium. For each measurement point $\boldsymbol{r}_{m}$ and angular frequency $\omega$, the measured scattered field $E_{S}$ is related to the unknown contrast function $\chi$ by the following linear integral equation:

$$
E_{s}\left(\boldsymbol{r}_{m}, \omega\right)=k_{b}^{2} \iint_{D} g_{e}\left(\boldsymbol{r}_{m}, \boldsymbol{r}, \omega\right) E_{i n c}(\boldsymbol{r}, \omega) \chi(\boldsymbol{r}) d \boldsymbol{r}
$$

where $k_{b}$ is the wave-number of the medium, $E_{\text {inc }}$ is the incident field in $D$ and $g_{e}$ is the Green's function accounting for the radiation at $\boldsymbol{r}_{m}$ by the elementary source located at $\boldsymbol{r}$. The inverse problem defined by (1) is solved by resorting to the adjoint inversion scheme [29]:

$$
\chi(\boldsymbol{r})=\iint\left(g_{e}\left(\boldsymbol{r}_{m}, \boldsymbol{r}, \omega\right) E_{\text {inc }}(\boldsymbol{r}, \omega)\right)^{*} E_{s}\left(\boldsymbol{r}_{m}, \omega\right) d \omega d \boldsymbol{r}_{m}
$$

which is essentially equivalent to a frequency-domain back projection [29].

The spatial map defined by the amplitude of the contrast function retrieved via (2) is referred to as a tomographic image. The presence of high contrast areas provides indications on the position and geometry of targets, e.g. decayed areas or tree hollows.

In regard to the present study, the raw radargrams are presented in time domain, subsequently processed and then focused with the adjoint inversion scheme in the frequency domain. The overall data processing framework consists in the following steps:

- zero-time setting;

- background removal to filter the direct coupling and the reflection from the air/trunk interface. To this end, the 
subspace projection method [31] is adopted. Specifically, the singular value decomposition (SVD) of each B-scan matrix is performed and the first $N_{t}$ terms of the singular spectrum, representative of the clutter, are filtered;

- $\quad$ Fourier transform of the filtered raw data in order to achieve the data in the frequency domain;

- adjoint inversion.

\section{RESUlTS AND SHORT DisCUSSION}

Data pre-processing was applied to the raw radargrams by setting the zero-time at 2.2 ns. Moreover, the SVD-based background removal procedure was implemented by filtering the first $N_{t}=10$ terms in the singular spectrum of each B-scan. Figure 2 shows a comparison between raw (Fig. 2a) and preprocessed (Fig. 2b) radargrams collected along the first profile $(h=0)$.

The presence of the tree hollow is identified in the processed data output by the signal reflections at a two-way travel time in a range until to about 5-6 ns.

After the pre-processing stage, the adjoint inversion approach was then applied by considering the frequency band 500-2000 MHz sampled with 31 frequencies uniformly spaced by $50 \mathrm{MHz}$. The trunk section was discretised into image pixels with size $1 \mathrm{~cm}$ and the relative dielectric permittivity was set at $\varepsilon=40$. Permittivity measurements were conducted using a 1 $\mathrm{GHz}$ horn antenna system from IDS GeoRadar (Part of Hexagon), in line with the methodology reported in [32].

The tomographic image in Fig. 3 refers to the tree section $h$ $=0 \mathrm{~m}$. Several anomalies can be observed in the wood area, whereas no targets are detected in the tree hollow. This result is consistent with the radargram in Fig. $2 \mathrm{~b}$, which shows a notable attenuation of the signal reflections after 6 ns. Regardless of these attenuation effects, it is important to note how the tomographic approach allows to focus those reflections at the location points where they originate.

Therefore, this method contributes to ease the interpretation of the internal configuration of the tree. Indeed, the microwave tomography approach is able to locate the tree hollow with a good accuracy and in a robust way.

\section{CONCLUSION}

This paper presents a demonstration of the groundpenetrating radar (GPR) capability in detecting hollows and cavities in tree trunks.

The data collection methodology as well as the hand-held GPR antenna system and central frequency $(2 \mathrm{GHz})$ used for the investigation have proven viable for use on hollow trees.

The application of a tomographic inversion approach was proven effective in detecting the main structural features of a hollow tree.

In particular, it is clear how the proposed approach can assess this particular tree types as well as other information such as the thickness of the solid structural layer.

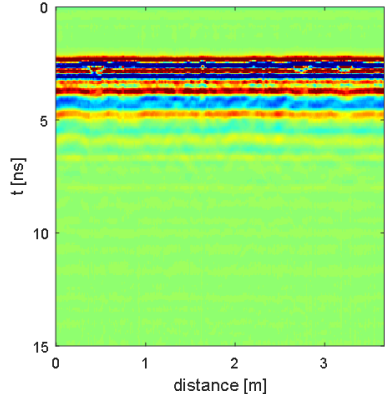

(a)

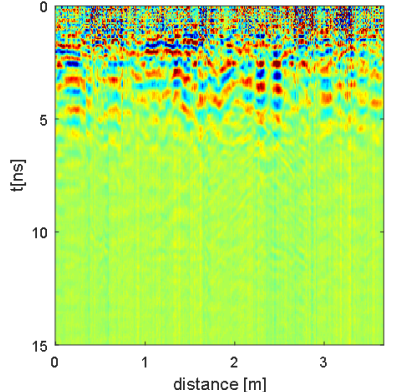

(b)
Fig. 2. Radargrams of the investigated tree at $h=0 \mathrm{~m}$. Raw radargam (a) and processed radargram after the application of the pre-processing stage (b).

Future research could task itself on the application of the tomographic approach to multi-frequency GPR data collected on hollow tree trunks, in order to explore whether a more comprehensive amount of information, in terms of decay types and their location, can be provided.
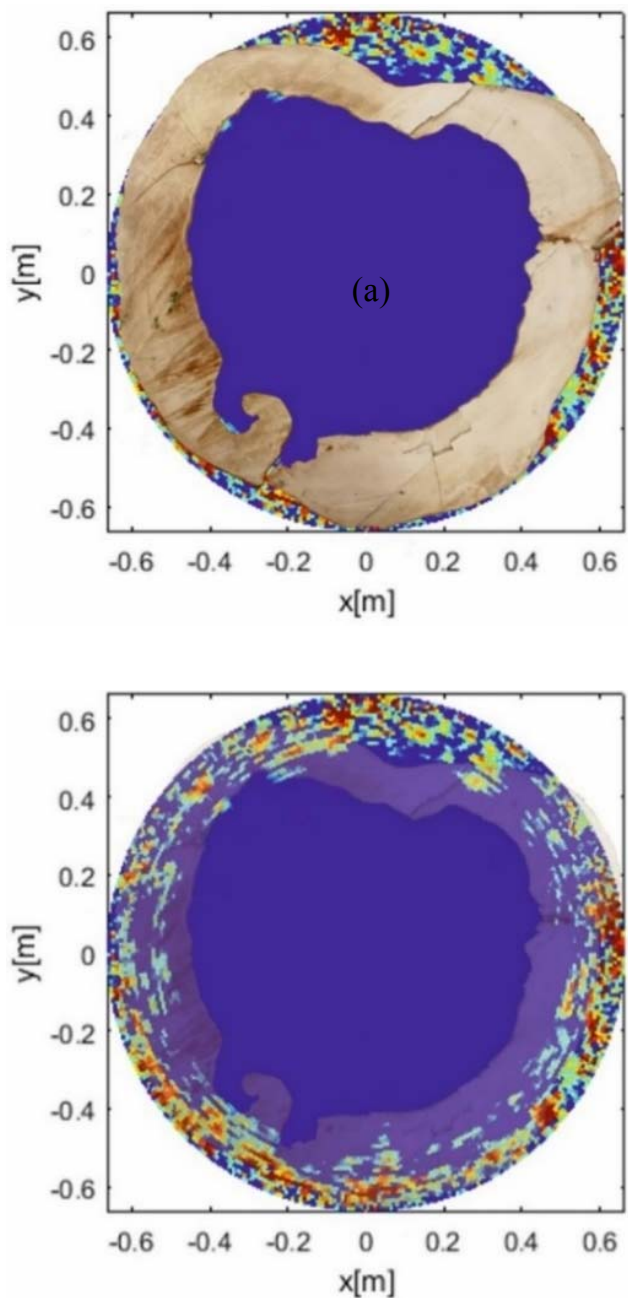

(b)

Fig. 3. Tomographic image of the tree section at $h=0 \mathrm{~m}$. (a) Planar view of the cut tree section, and (b) superimposition with the spatial map, representing the contrast function of the trunk section. 
Realistic numerical simulations could be developed based on the real-life information (i.e., the internal structure of the tree as well as the location and size of the internal decay) collected on the tree as well as the electromagnetic data gathered from the inspections. These developments could contribute to expand the existing knowledge in terms of the tomography applications in this area of endeavour.

\section{REFERENCES}

[1] P. K. Anderson, A. A. Cunningham, N. G. Patel, F. J. Morales, P. R. Epstein and P. Daszak, "Emerging infectious diseases of plants: pathogen pollution, climate change and agrotechnology drivers," Trends in ecology \& evolution, vol. 19, no. 10, pp. 535-544, 2004.

[2] Z. Zheng, S. Zhang, C. Baskin, J. Baskin, D. Schaefer, X. Yang and L. Yang, "Hollows in living trees develop slowly but considerably influence the estimate of forest biomass," Functional Ecology, vol. 30, no. 5, pp. 830-838, 2016

[3] H. M. Good, J. T. Basham and S. D. Kadzielawa, "Respiratory activity of fungal associations in zones of heart rot and stain in sugar maple," Canadian Journal of Botany, vol. 46, no. 1, pp. 27-36, 1968.

[4] K. L. Cockle, K. Martin and G. Robledo, "Linking fungi, trees, and holeusing birds in a Neotropical tree-cavity network: Pathways of cavity production and implications for conservation," Forest Ecology and Management, vol. 264, pp. 210-219, 2012.

[5] D. B. Clark and D. A. Clark, " Landscape-scale variation in forest structure and biomass in a tropical rain forest," Forest ecology and management, vol. 137, no. 1-3, pp. 185-198, 2000.

[6] S. G. Pallardy, Physiology of woody plants, Academic Press, 2010.

[7] A. B. Edworthy and K. Martin, "Long-term dynamics of the characteristics of tree cavities used for nesting by vertebrates," Forest ecology and management, vol. 334, pp. 122-128, 2014.

[8] C. Mattheck, K. Bethge and P. W. West, "Breakage of hollow tree stems," Trees, vol. 9, no. 1, pp. 47-50, 1994.

[9] M. J. Ellison, "Quantified tree risk assessment used in the management of amenity trees," Journal of Arboriculture, vol. 31, no. 2, pp. 57-65, 2005.

[10] S. Denman, N. Brown, S. Kirk, M. Jeger and J. Webber, "A description of the symptoms of Acute Oak Decline in Britain and a comparative review on causes of similar disorders on oak in Europe," Forestry: An International Journal of Forest Research, vol. 87, no. 4, pp. 535-551, 2014.

[11] G. Deflorio, S. Fink and F. W. Schwarze, "Detection of incipient decay in tree stems with sonic tomography after wounding and fungal inoculation," Wood Science and Technology, vol. 42, no. 2, pp. 117-132, 2008.

[12] S. A. Al Hagrey, "Electrical resistivity imaging of tree trunks," Near Surface Geophysics, vol. 4, no. 3, pp. 179-187, 2006.

[13] Q. Wei, B. Leblon and A. La Rocque, "On the use of X-ray computed tomography for determining wood properties: a review," Canadian journal of forest research, vol. 41, no. 11, pp. 2120-2140, 2011.

[14] A. Catena, "Thermography shows damaged tissue and cavities present in trees," Nondestructive Characterization of Materials, pp. 515-524, 2003.

[15] F. Boero, A. Fedeli, M. Lanini, M. Maffongelli, R. Monleone, M. Pastorino, A. Randazzo, A. Salvadè and A. Sansalone, " Microwave tomography for the inspection of wood materials: Imaging system and experimental results," IEEE Transactions on Microwave Theory and Techniques, vol. 66, no. 7, pp. 3497-3510, 2018.

[16] A. M. Alani, F. Soldovieri, G. Gennarelli, I. Giannakis, I. Catapano, L. Lantini, G. Ludeno and F. Tosti, "A tomographic inversion approach for the detection of decay and cavities in tree trunks using ground penetrating radar," in 10th International Workshop on Advanced Ground Penetrating Radar, The Hague, The Netherlands, 2019.
[17] A. M. Alani, F. Soldovieri, I. Catapano, I. Giannakis, G. Gennarelli, L. Lantini, G. Ludeno and F. Tosti, "The Use of Ground Penetrating Radar and Microwave Tomography for the Detection of Decay and Cavities in Tree Trunks," Remote Sensing, vol. 11, no. 18, 2019.

[18] H. Lorenzo, V. Pérez-Gracia, A. Novo and J. Armesto, "Forestry applications of ground-penetrating radar," Forest Systems, vol. 19, no. 1, pp. 5-17, 2010.

[19] J. Ježová, L. Mertens and S. Lambot, "Ground-penetrating radar for observing tree trunks and other cylindrical objects," Construction and Building Materials, vol. 123, pp. 214-225, 2016.

[20] A. M. Alani, L. B. Ciampoli, L. Lantini, F. Tosti and A. Benedetto, "Mapping the root system of matured trees using ground penetrating radar," in 2018 17th International Conference on Ground Penetrating Radar (GPR), Rapperswil, Switzerland, 2018.

[21] L. Lantini, R. Holleworth, D. Egyir, I. Giannakis, F. Tosti and A. Alani, "Use of ground penetrating radar for assessing interconnections between root systems of different matured tree species," in 2018 IEEE International Conference on Metrology for Archaeology and Cultural Heritage, Cassino, Italy, 2018.

[22] A. M. Alani and L. Lantini, "Recent advances in tree root mapping and assessment using non-destructive testing methods: a focus on ground penetrating radar," Surveys in Geophysics, pp. 1-42, 2019.

[23] L. Lantini, F. Tosti, I. Giannakis, D. Egyir, A. Benedetto and A. M. Alani, "A Novel Processing Framework for Tree Root Mapping and Density Estimation using Ground Penetrating Radar," in 10th International Workshop on Advanced Ground Penetrating Radar, The Hague, The Netherlands, 2019.

[24] G. Leucci, N. Masini, R. Persico and F. Soldovieri, "GPR and sonic tomography for structural restoration: the case of the cathedral of Tricarico," Journal of Geophysics and Engineering, vol. 8, no. 3, pp. S76S92, 2011.

[25] L. L. Monte, D. Erricolo, F. Soldovieri and M. C. Wicks, "Radio frequency tomography for tunnel detection," IEEE Transactions on Geoscience and Remote Sensing, vol. 48, no. 3, pp. 1128-1137, 2009.

[26] I. Giannakis, F. Tosti, L. Lantini and A. M. Alani, "Health Monitoring of Tree Trunks Using Ground Penetrating Radar," IEEE Transactions on Geoscience and Remote Sensing, vol. 57, no. 10, pp. 8317-8326, 2019.

[27] I. Giannakis, F. Tosti, L. Lantini and A. M. Alani, "Diagnosing emerging infectious diseases of trees using ground penetrating radar," IEEE Transactions on Geoscience and Remote Sensing, vol. 58, no. 2, pp. 1146$1155,2019$.

[28] F. Soldovieri and R. Solimene, "Ground Penetrating Radar Subsurface Imaging of Buried Objects," in Radar Technology, In-Tech, 2010, pp. 105-126.

[29] R. Solimene, I. Catapano, G. Gennarelli, A. Cuccaro, A. Dell'Aversano and F. Soldovieri, "SAR Imaging Algorithms and Some Unconventional Applications: A unified mathematical overview," IEEE Signal Processing Magazine, vol. 31, no. 4, pp. 90-98, 2014.

[30] I. Catapano, G. Gennarelli, G. Ludeno, F. Soldovieri and R. Persico, "Ground Penetrating Radar: Operation Principle and Data Processing," Wiley Encyclopedia of Electrical and Electronics Engineering, pp. 1-23, 1999 .

[31] R. Solimene, A. Cuccaro, A. Dell'Aversano, I. Catapano, and F. Soldovieri, "Ground clutter removal in GPR surveys," IEEE Journal of Selected Topics in Applied Earth Observations and Remote Sensing, vol. 7, no. 3, pp. 792-798, 2013.

[32] A. M. Alani, I. Giannakis, L. Zou, L. Lantini and F. Tosti, "Reverse-time migration for evaluating the internal structure of tree-trunks using groundpenetrating radar," NDT\&E International, accepted (in press). 